\title{
Identification, design and kinematic analysis of an earthmoving mechanism.
}

\author{
Yongjun Pan, Alfonso Callejo
}

\begin{abstract}
Earthmoving mechanisms in motor graders are critical components for earthwork, compaction and re handling, and yet they have not received much attention by mechanical engineering research in recent times. In this paper, a comprehensive analysis, from mechanism identification and innovative design to kinematic analysis, is presented. First, the mechanism analysis and synthesis method based on multibody system dynamics is carried out through the analysis of the system topology and connectivity. We conclude that the earthmov ing multibody system is a spatial hybrid mechanism, which consists of a spatial parallel mechanism and a spatial serial mechanism. Second, a number of new spatial parallel mechanisms, which are advantageous with respect to the original one under certain conditions, are generated. The kinematic characteristics of the parallel mechanism family are investigated in terms of constraint equations formu Jated in natural coordinates. Third and last, kinematic simulations and optimization processes are carried out to evaluate the advantages of the presented spatial parallel mechanisms. Simulation results show that these mechanisms can provide better kinematic performance.
\end{abstract}

Keywords Motor grader; Earthrnoving mechanism; Multibody systems; 3RRPS S mechanism; Natural coordinates

\section{Introduction}

Motor graders are pieces of construction machinery usually eq uipped with a blade operation device. They can be used to prepare a wide flat surface, set native soil foundation pads, prod uce inclin ed surfaces, and provide drainage ditches with shallow V-shaped cross sections in construction, mining, agriculture and military engineering. An overview of an example motor grader is shown in Fig. 1.

The earthmoving mechanism is a key component of motor graders. A typical structure of an earthmoving mechanism is shown in Fig. 2. It operates directly with earth and plays a crucial role d uring earthwork, com- paction and re-handling. The mechanism can be considered as a complex multibody system (MBS). In order to ensure rigid construction, simple operation and efficient performance, innovative design and kinematic and dynarnic analyses can be used.

The earthmoving mechanism consists of an overhead frame, a swing frame, a traction frame, a turntable, severa! rod forks and severa! blade positioning cylinders. The blade yaw angle is commanded by a su bsystem containing components with relative translations and rotations. The overhead frame is a large-scale, box-type welding part, which bears heavy loads resulting from the imposed joint constraints. As can be seen in Fig. 2, the swing frame connects the overhead frame arms through axis pins, which 


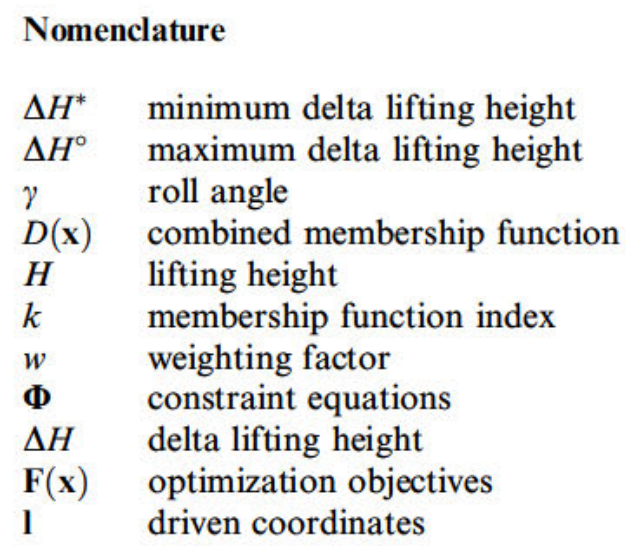

$\begin{array}{ll}\mathbf{q} & \text { generalized coordinates } \\ \mathbf{r} & \text { Cartesian positions } \\ \mathbf{u} & \text { Cartesian components of unit vectors } \\ \mathbf{x} & \text { design parameters } \\ H^{*} & \text { maximum lifting height } \\ H^{\circ} & \text { minimum lifting height } \\ N(\mathbf{x}) & \text { membership function } \\ s & \text { known distance depending on points } \\ \mathbf{\Phi}_{t} & \text { constraint equations partial derivative } \\ \boldsymbol{\Phi}_{\mathbf{q}} & \text { Jacobian matrix }\end{array}$

q generalized coordinates

r Cartesian positions

u Cartesian components of unit vectors

$\mathbf{x}$ design parameters

$H^{*} \quad$ maximum lifting height

$H^{\circ} \quad$ minimum lifting height

$N(\mathbf{x}) \quad$ membership function

$\boldsymbol{\Phi}_{t} \quad$ constraint equations partial derivative

$\boldsymbol{\Phi}_{\mathrm{q}} \quad$ Jacobian matrix means that the relative position is adjusted before operations according to work conditions. Hence, they can be modeled as a single fixed part during system operation.

Rod forks are relatively small components. They bear high forces coming from the swing frame and cylinders. In fact, fatigue cracks or failure in the rod fork are some of the most common mechanism failures according to customer feedback questionnaires. Hydraulic cylinders connect the overhead frame to the traction frame, and they are used to lift the blade and rotate it. The traction frame is a welding part and works as a moving platform to lift and rotate the blade and propagate traction forces.

Finally, the turntable has internal teeth and an internal motor so that it can rotate with respect to the traction frame. It also supports the subsystem that adjusts the cutting angle and lateral motion. This subsystem contains a blade, two angle regulators, and several fasteners and cylinders. The motor inside the turntable controls the blade yaw angle around the vehicle's vertical axis.

Research on the kinematics and dynamics of earthmoving equipment, especially within hydraulic excavators and wheel loaders, has received significant attention recently. In most cases, it is very difficult or even impossible to determine dynamic solutions using analytical methods. Furthermore, real tests on physical prototypes are too laborious and pricey. Consequently, numerical simulations are often regarded as better alternatives to solve the dynamic problems.
Hemami (1992, 1993) introduced an analysis and modeling technique for the automatic scooping and loading of a loader excavating machine and discussed the optimal motion of a loader bucket. Vähä and Skibniewski (1993) described a dynamic model of an excavator based on Newton Euler's equations in a local coordinate frame. Koivo (1994, 1996) presented a dynamic model and a systematic procedure to obtain an excavator's kinematic and dynamic performance. Fox et al. (2002) developed a dynamic model of digging operations based on a multibody system approach. Towarek (2003) discussed the dynamics of a spatial model of a single-bucket excavator considering the strain of soil foundation. Takahashi et al. (2004) proposed a concept of advanced load-haul-dump with a vessel (ALV) and described its kinematics and dynamics. Frimpong et al. (2005), Frimpong and Li (2005), Li and Frimpong (2008) and Frimpong et al. (2008) developed a dynamics model and a hybrid virtual prototype toward an intelligent shovel excavator, and they also analyzed its dynamic characteristics. Fales and Kelkar (2009) introduced two $\mathbf{H}_{\infty}$-based robust control designs for a wheel loader bucket leveling mechanism, on the basis of a multi-input, multi-output nonlinear linkage model. Chen et al. (2014) described an optimal method for backhoe excavators including digging paths.

Based on the known dynamic characteristics of earthmoving equipment, some researchers have also presented finite element and fatigue analyses to calculate structural

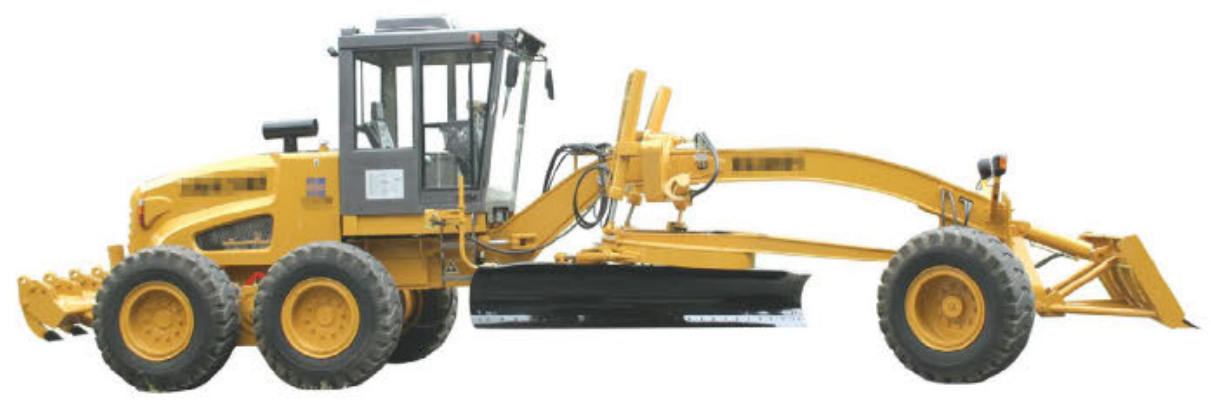

Fig. 1. Motor grader overview. 


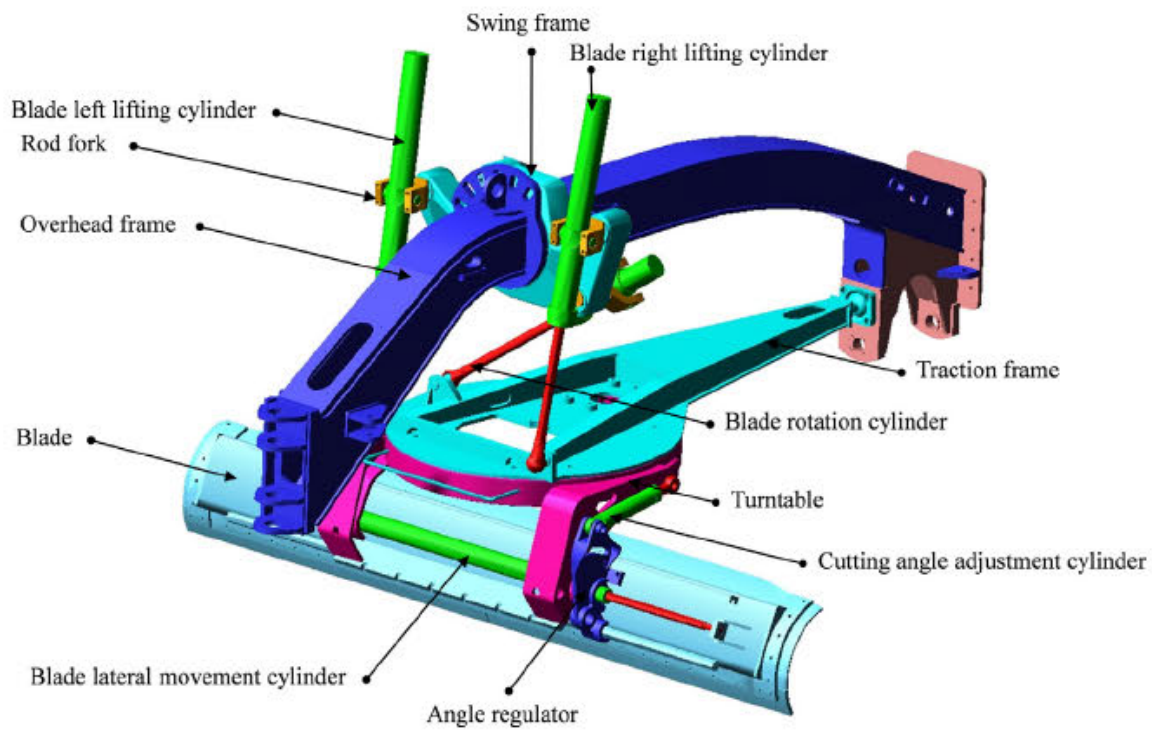

Fig. 2. Earthmoving mechanism structure.

strength requirements. Rusiński et al. (2008) investigated cracked booms in underground mining machines, for which they employed the finite element method. Jia et al. (2013) introduced a modeling framework for a load haul dump, which integrated product design, multibody dynamics, finite element and fatigue analyses. Frimpong and $\mathrm{Li}$ (2005) and Li and Frimpong (2008) presented dynamics and finite element models for a shovel excavator based on ADAMS and NASTRAN software packages, respectively. Seungjin et al. (2010) and Kim et al. (2013) developed an analytic gradient-based optimization algorithm to perform optimal design of hydraulic excavators. Shen et al. (2013) established a comprehensive set of genetic algorithms to optimize the mathematical model of a loader device.

In our opinion, much of the work in the literature has dealt with the kinematics and dynamics of hydraulic excavators and wheel loaders. On the other hand, very few references on the kinematic and dynamic performance of earthmoving equipment can be found in the literature. The earthmoving mechanism of motor graders is somewhat difficult to investigate since it not only bears high external forces and vibrations, but also involves very different positions and orientations. Sobczyk and Tora (1998) introduced a blade stabilization system to compensate the grading errors caused by the vertical displacement of the motor grader. Carmen and Silviu (2012) evaluated the operational performances for motor grader earthmoving equipment with different structural and functional configurations. Zhen et al. (2011) provided solutions to increase the operator's comfort by decreasing noise.

This paper introduces a method for the systematic synthesis, innovative design, and kinematic analysis of the earthmoving mechanism of a motor grader. Natural coordinates are used to formulate kinematic constraint equations in a simple way. We provide a number of original and effective designs through identification and innovative design. Their kinematic performance and effectiveness is theoretically studied and backed with kinematic simulations. Furthermore, the working performance of an earthmoving mechanism is improved through design and optimization, instead of through expensive hydraulic actuation or position sensors. This approach can be applied to all kinds of earthmoving equipment, from common heavy machines to unusual machines such as motor graders.

\section{Mechanism identification}

In order to carry out a thorough mechanism analysis and synthesis, a virtual prototyping model (described in Fig. 2) is considered as an example. The earthmoving system has 22 bodies, 27 joints and 6 degrees of freedom (DOF) corresponding to the 6 blade driving cylinders ( 2 lifting cylinders, 1 roll rotation cylinder, 1 yaw rotation cylinder, 1 cutting angle adjustment cylinder and 1 lateral translation cylinder). The multibody system topology is depicted in Fig. 3.

The overhead frame, which is assumed to be fixed to the ground, is connected to the traction frame through a spherical joint at the front of the mechanism. The swing frame, which is fixed to the overhead frame during machine operation, is connected to the rod forks via three revolute joints. Two blade lifting cylinders and one blade roll cylinder are connected to the rod forks through three revolute joints on top of the mechanism and are attached to the traction frame via three spherical joints at the bottom. The turntable and traction frame are connected through a revolute joint, which is driven by a motor inside the turntable. The cutting angle adjustment cylinders are linked to the turntable on one side and to the angle regulators on the other side. The angle regulators are attached to the turntable through revolute joints and to the blade lateral cylinder 


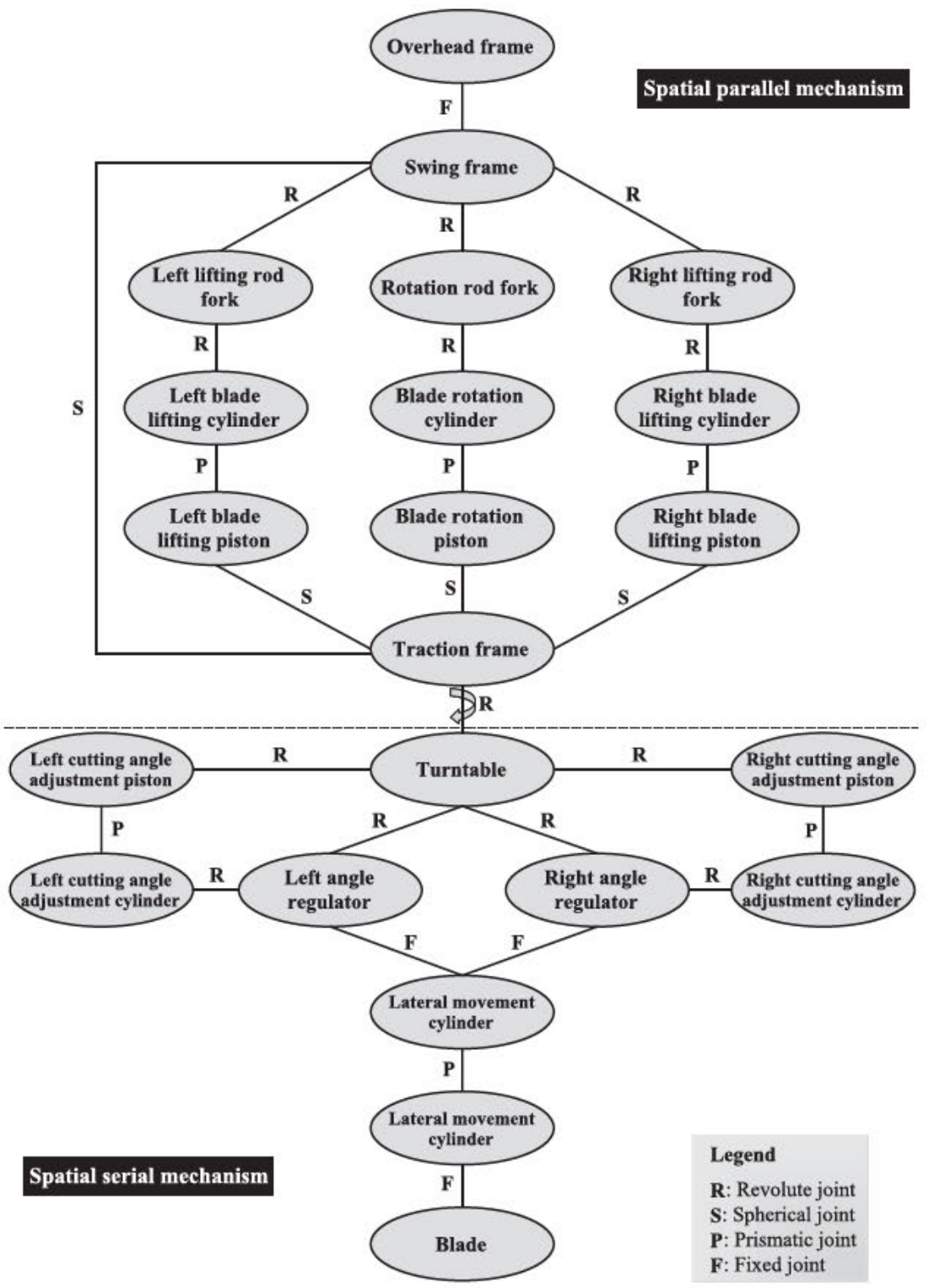

Fig. 3. Earthmoving mechanism topology.

Table 1

Operations of driven cylinders.

\begin{tabular}{llll}
\hline Driven motions & Operations & & \\
\hline Blade lateral translation & $\rightarrow$ & & \\
Cutting angle adjustment & & $\rightarrow$ & \\
Blade yaw rotation & & & \\
Left/right blade lifting & & & $\rightarrow$ \\
Blade roll rotation & & & $\rightarrow$ \\
\hline
\end{tabular}

through fixed joints. The blade is connected to the blade lateral cylinder piston. A closed-loop topology results from these kinematic connections. The six hydraulic cylinders are driven as shown in Table 1, where the arrow represents the corresponding cylinder driving operation. In our opinion, this earthmoving mechanism is one of the most important applications of MBS dynamics within construction and heavy machinery.
Based on the system topology and cylinder operation, the system can be decomposed into two subsystems (described in Fig. 3): a spatial parallel mechanism and a spatial serial mechanism. The spatial parallel mechanism is described in Fig. 4(a). It consists of four subchains, namely three revolute-revolute-prismatic-spherical (RRPS) subchains and one spherical subchain connecting the overhead frame and traction frame from the top to the bottom. Hence, this subsystem is a 3RRPS-S mechanism.

The spatial parallel mechanism comprises three independent, driven motions which result from the blade lifting and roll rotation cylinders. It is possible to calculate the DOF of this spatial parallel mechanism as shown in Table 2. Zero DOF result from this count, which allows one to easily perform kinematic analyses. This parallel mechanism has some unique characteristics when compared to serial mechanisms: a more rigid structure, high 


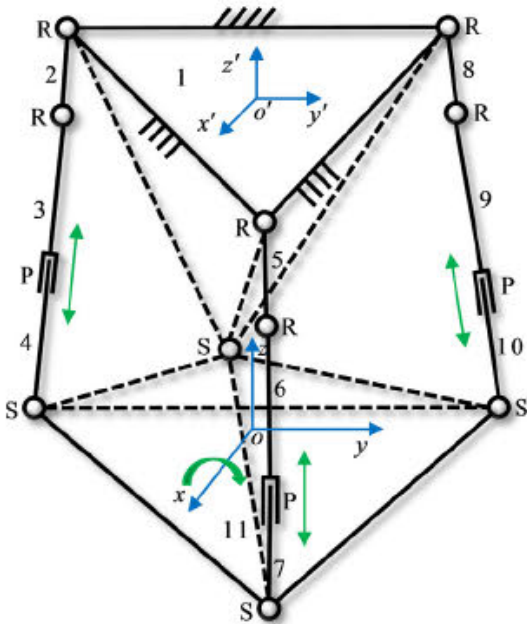

Legend:

(a) 3RRPS-S mechanism

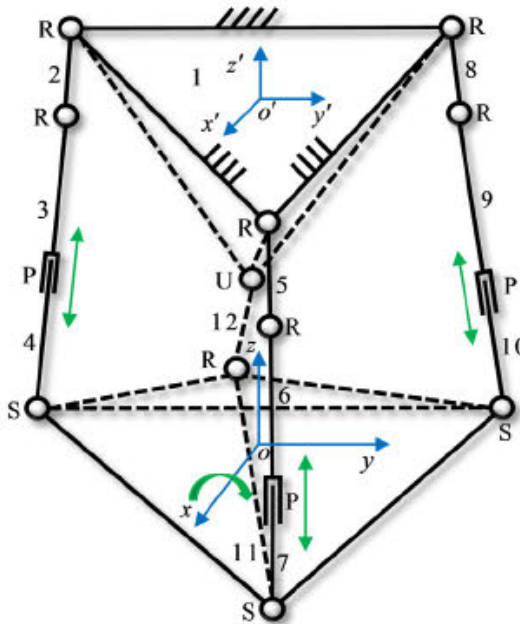

(b) 3RRPS-UR mechanism

9: Right blade lifting cylinder 10: Right blade lifting piston

11: Traction frame

12: Lower transmission piece

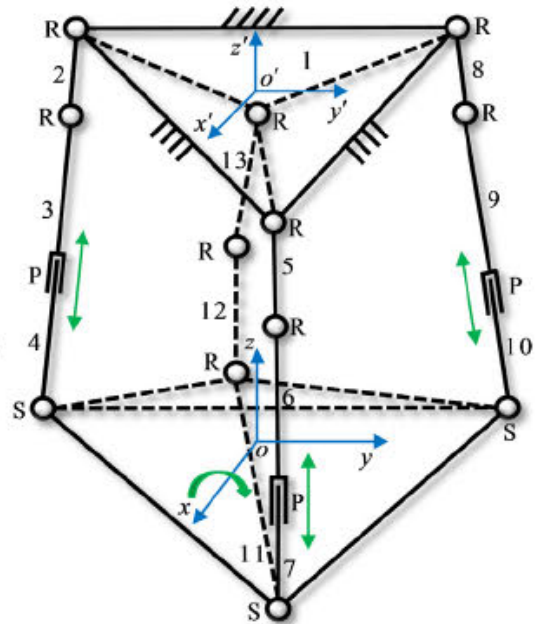

(c) 3RRPS-RRR mechanism

13: Upper transmission piece

$\mathrm{R}$ : Revolute joint

P: Prismatic joint

S: Spherical joint

Fig. 4. 3 DOF spatial parallel mechanisms.

Table 2

Degrees of freedom of the 3RRPS S mechanism.

\begin{tabular}{lccc}
\hline Components & Number & DOF & IDOF \\
\hline Parts & 10 & 6 & 60 \\
Prismatic & 3 & 5 & 15 \\
Revolute & 6 & 5 & 30 \\
Spherical & 4 & 3 & 12 \\
Motions & 3 & 1 & 3 \\
& & & $\Sigma$ DOF $=0$ \\
\hline
\end{tabular}

positioning precision, high acceleration capabilities and stable operation. Similar 3-DOF spatial parallel mechanisms have been studied by researchers in terms of mechanisms, kinematics and dynamics (Staicu et al., 2007; Staicu, 2009; Wu et al., 2009, 2013; Li et al., 2010; Yen and Lai, 2009).

On the other hand, the spatial serial mechanism consists of three planar serial mechanisms. Two of them are located between the turntable and left and right angle regulators to adjust the blade cutting angle, and the third planar mechanism operates between the angle regulators and the blade to control the blade lateral translation. This spatial serial mechanism is over-constrained due to the same structure in both left and right side for cutting angle adjustment. It has two DOF corresponding to the blade lateral translation and the cutting angle adjustment. We do not further discuss the performance of this mechanism in the paper.

Both the spatial parallel and serial mechanisms make up the earthmoving system, and they are assembled through a revolute joint coming from a gear rack transmission inside the turntable. In conclusion, the earthmoving MBS is a 6DOF spatial hybrid mechanism and the 3RRPS-S spatial parallel mechanism is one of its crucial subsystems (Choi et al., 2005).

\section{Mechanism design}

Innovation is widely considered to be essential during engineering design. In this section, innovative design is carried out to derive new structures that may be suitable for special operation conditions. The 3RRPS-S mechanism is used as a reference to generate different mechanisms.

The fracture of the spherical joint between the overhead and traction frames is a common fatigue failure mode. Once it happens, it is very inconvenient to fix. The maintenance cost is usually high because the overhead and traction frames, which are large-scale components with complex structure, have to be replaced or fully fixed.

As part of a robust design improvement, a small-scale rod is introduced to prevent the fracture from happening in the traction and overhead frames. This means that, by carrying out appropriate structural design (which is out of the scope of this paper), the failure will always take place in the small-scale rod. This is an affordable, fast and efficient fix. Fig. 4(b) shows the derivative mechanism. It has three revolute-revolute-prismatic-spherical subchains and one universal-revolute subchain, and its DOF count is shown in Table 3. Note that the original spherical subchain is replaced by a universal-revolute subchain through the

Table 3

Degrees of freedom of the 3UPS UR mechanism.

\begin{tabular}{lccc}
\hline Components & Number & DOF & $\Sigma$ DOF \\
\hline Parts & 11 & 6 & 66 \\
Prismatic & 3 & 5 & 15 \\
Revolute & 7 & 5 & 35 \\
Universal & 1 & 4 & 4 \\
Spherical & 3 & 3 & 9
\end{tabular}

$\Sigma \mathrm{DOF}=3$ 
introduction of one rod. Thus, it can be regarded as a 3DOF 3RRPS-UR mechanism.

Analogously, upper and lower rods can be introduced in the original spatial parallel mechanism so as to connect the overhead and traction frames. The new mechanism is depicted in Fig. 4(c). This parallel mechanism allows for more design freedom because two parametric rods have been introduced. It has three revolute-revolute-prismatic-s pherical subchains and one revolute-revolute-revolute subchain. Its DOF count is shown in Table 4. Note that the original spherical subchain is replaced by a revoluterevolute-revolute subchain through the introduction of two rods. Thus, it can be concluded that it is a $3-\mathrm{DOF}$ 3RRPS-RRR mechanism.

The presented derivative mechanisms, together with the original one, constitute a family of 3-DOF spatial parallel mechanisms with four subchains, which may be suitable for earthmoving MBS in different situations. Their kinematic and dynamic characteristics ought to be investigated to determine in which conditions they are advantageous. Next, we carry out kinematic analyses to assess their performance.

\section{Kinematic analysis}

In this section, we consider the 3RRPS-S mechanism as an example to construct the kinematic equations using MBS theory. Specifically, natural coordinates (Cartesian coordinates of basic points and Cartesian components of unit vectors García de Jalón et al., 1986; García de Jalón, 2007; García de Jalón and Callejo, 2011) are used to describe the position of each rigid body. We consider the overhead frame as a fixed platform with three revolute joints located at fixed points. On the other hand, we consider the traction frame as a moving platform with four spherical joints. One of those connects the overhead frame to the traction frame, and the others, which are movable, connect the hydraulic cylinders to the traction frame. Next, the kinematic characteristics of these three moving spherical joints, which are chosen to represent the performance of this parallel mechanism, are theoretically investigated.

Fig. 5 depicts the 3RRPS-S parallel mechanism modeled with natural coordinates. This mechanism has 9 moving points, 6 moving unit vectors and 3 driven coordinates corresponding to the blade lifting and rotation cylinders, for a total of 45 dependent Cartesian coordinates. Let us define the vector of generalized coordinates as:

Table 4

Degrees of freedom of the 3RRPS RRR mechanism.

\begin{tabular}{lccc}
\hline Components & Number & DOF & $\Sigma$ DOF \\
\hline Parts & 12 & 6 & 72 \\
Prismatic & 3 & 5 & 15 \\
Revolute & 9 & 5 & 45 \\
Spherical & 3 & 3 & 9
\end{tabular}

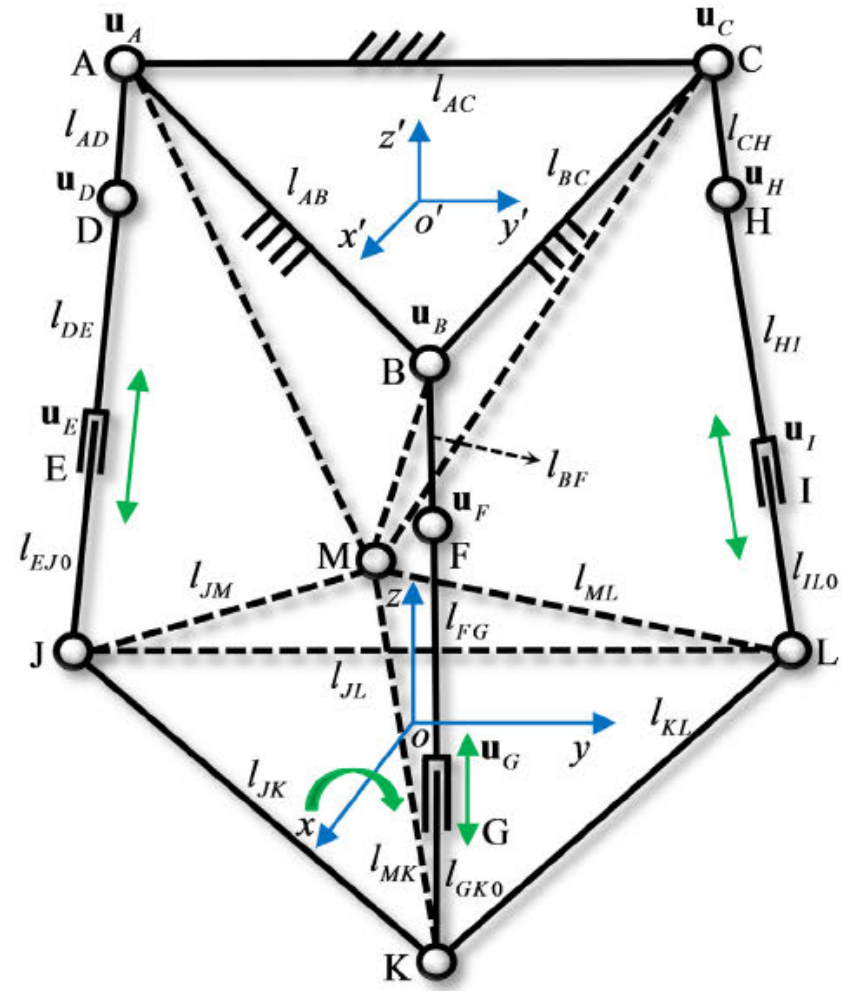

Fig. 5. 3RRPS $\mathrm{S}$ mechanism modeled with natural coordinates.

$\mathbf{q}=\left\{\mathbf{r}_{D}^{T}, \mathbf{u}_{D}^{T}, \mathbf{r}_{E}^{T}, \mathbf{u}_{E}^{T}, \mathbf{r}_{J}^{T}, \mathbf{r}_{F}^{T}, \mathbf{u}_{F}^{T}, \mathbf{r}_{G}^{T}, \mathbf{u}_{G}^{T}, \mathbf{r}_{K}^{T}, \mathbf{r}_{H}^{T}, \mathbf{u}_{H}^{T}, \mathbf{r}_{I}^{T}, \mathbf{u}_{I}^{T}, \mathbf{r}_{L}^{T}\right\}^{T}$

where vectors $\mathbf{r}$ and $\mathbf{u}$ represent, respectively, Cartesian positions and Cartesian components of unit vectors:

$\mathbf{r}=\{x, y, z\}^{T}, \quad \mathbf{u}=\left\{u_{x}, u_{y}, u_{z}\right\}^{T}$

The driven coordinates of the blade lifting and rotation cylinders are expressed as:

$\mathbf{I}=\left\{l_{E J}, l_{G K}, l_{I L}\right\}^{T}$

where scalar $l$ denotes the distance of the driven cylinders as a function of time, which takes the following form:

$l_{E J}=f(t), \quad l_{G K}=g(t), \quad l_{I L}=h(t)$

Mathematically, the kinematic problem can be reduced to determine the vector of dependent coordinates that satisfies the system of nonlinear kinematic constraint equations from the (known) cylinder coordinates. The constraint equations can be easily constructed following the natural coordinate formulation (García de Jalón and Callejo, 2011). Table 5 shows the constraint equations corresponding to the left lifting, rotation and right lifting subchains.

Each RRPS subchain consists of rigid body constraints and joint constraints. The first ten equations describe the rigid body constraint conditions. The last three equations correspond to the prismatic joint, where the first two equations ensure that vector $\mathbf{r}_{J E}$ is aligned with unit vector $\mathbf{u}_{E}$, and the last equation guarantees that bodies $D E$ and $E J$ 
Table 5

Constraint equations of the 3RRPS S mechanism.

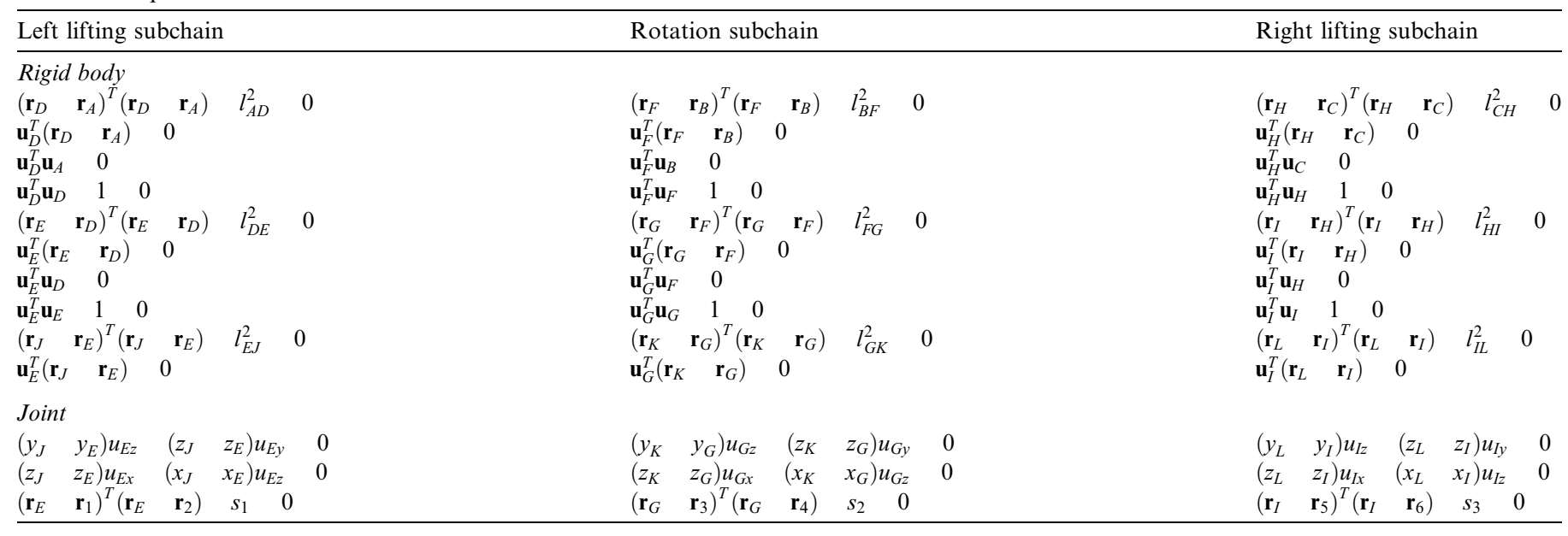

cannot rotate with respect to each other. Points $\mathbf{r}_{1}, \mathbf{r}_{2}, \mathbf{r}_{3}, \mathbf{r}_{4}, \mathbf{r}_{5}$ and $\mathbf{r}_{6}$ are in bodies $D E, E J, G F, K G, I H$ and $L I$, and $s_{1}, s_{2}$, and $s_{3}$ are scalars depending on the chosen Cartesian points in each subchain. In order to enforce the last prismatic joint equation, the Cartesian points have to be carefully selected so that they are non-collinear with the joint's axis and they do not form an angle close to zero.

The moving platform has to meet a number of constant distance constraints:

$$
\begin{aligned}
& \left(\mathbf{r}_{K}-\mathbf{r}_{J}\right)^{T}\left(\mathbf{r}_{K}-\mathbf{r}_{J}\right)-l_{J K}^{2}=0 \\
& \left(\mathbf{r}_{L}-\mathbf{r}_{K}\right)^{T}\left(\mathbf{r}_{L}-\mathbf{r}_{K}\right)-l_{K L}^{2}=0 \\
& \left(\mathbf{r}_{M}-\mathbf{r}_{L}\right)^{T}\left(\mathbf{r}_{M}-\mathbf{r}_{L}\right)-l_{L M}^{2}=0 \\
& \left(\mathbf{r}_{J}-\mathbf{r}_{M}\right)^{T}\left(\mathbf{r}_{J}-\mathbf{r}_{M}\right)-l_{M J}^{2}=0 \\
& \left(\mathbf{r}_{L}-\mathbf{r}_{J}\right)^{T}\left(\mathbf{r}_{L}-\mathbf{r}_{J}\right)-l_{J L}^{2}=0 \\
& \left(\mathbf{r}_{M}-\mathbf{r}_{K}\right)^{T}\left(\mathbf{r}_{M}-\mathbf{r}_{K}\right)-l_{K M}^{2}=0
\end{aligned}
$$

Combining all rigid body and joint constraint equations (Table 5 and Eqs. (5) (10)), 45 algebraic equations are obtained for the determination of the 45 dependent coordinates:

$\boldsymbol{\Phi}(\mathbf{q}, t)=\mathbf{0}$

Note that, in this example, the constraints are independent, holonomic and scleronomic, but are considered rheonomous for the sake of generality. In order to solve the system of nonlinear equations, the Newton Raphson method is implemented, starting from initial values. If $\mathbf{q}_{i}$ is an approximate position vector, we can linearize Eq. (11) as:

$\boldsymbol{\Phi}(\mathbf{q}, t) \cong \boldsymbol{\Phi}\left(\mathbf{q}_{i}\right)+\boldsymbol{\Phi}_{\mathbf{q}}\left(\mathbf{q}_{i}\right)\left(\mathbf{q}-\mathbf{q}_{i}\right)=\mathbf{0}$

where $\boldsymbol{\Phi}_{\mathbf{q}}$ denotes the Jocobian matrix of constraint equations. The iterative procedure to find $\mathbf{q}$ can be expressed as:

$\boldsymbol{\Phi}\left(\mathbf{q}_{i}\right)+\boldsymbol{\Phi}_{\mathbf{q}}\left(\mathbf{q}_{i}\right)\left(\mathbf{q}_{i+1}-\mathbf{q}_{i}\right)=\mathbf{0}$

The equations that allow one to solve the velocity and acceleration problems can be obtained, respectively, by differentiating Eq. (11) once and twice with respect to time:

$$
\begin{aligned}
& \boldsymbol{\Phi}_{\mathbf{q}}(\mathbf{q}, t) \dot{\mathbf{q}}=-\boldsymbol{\Phi}_{t} \\
& \boldsymbol{\Phi}_{\mathbf{q}}(\mathbf{q}, t) \ddot{\mathbf{q}}=-\dot{\boldsymbol{\Phi}}_{t}-\dot{\boldsymbol{\Phi}}_{\mathbf{q}} \dot{\mathbf{q}}
\end{aligned}
$$

where $\boldsymbol{\Phi}_{t}$ denotes the partial derivative of the constraint equations with respect to time. These two equations enable the computation of Cartesian velocities and accelerations starting from the initial positions and velocities. Similarly, the kinematic equations of the 3RRPS-UR and 3RRPSRRR mechanisms can be constructed by taking into account the constraint equations of the UR and RRR subchains.

\section{Optimization results}

The earthmoving mechanism is based on a number of fixed and moving pivots. The main purpose of the earthmoving mechanism design is to find appropriate moving pivots for efficient earthwork. Normally, the lifting height, parallel lifting capability and roll angle are key performance indicators (KPIs) of earthmoving equipment. These are often used to represent the trafficability, screeding and scraping capabilities (Xu and Huang, 1985). Even though other KPIs such as blade length, lateral movement length, cutting angle and yaw angle could be considered, they would not be affected by the earthmoving parallel mechanism pivots and they would not be modified during the mechanism optimization process.

In order to assess the advantages of the 3RRPS-UR and 3RRPS-RRR designs, the scraping, lifting and parallel lifting capabilities of these two mechanisms are analyzed and compared with the original 3RRPS-S mechanism. The initial location and orientation of joints in the original mechanism are shown in Table 6. By driving the three hydraulic cylinders to accomplish blade rotation and lifting, the kinematic analysis of the 3-DOF spatial parallel mechanism family can be carried out. To that end, Eqs. (11), (14), and (15) are solved once per driving position, velocity and acceleration. This results in the Cartesian positions, velocities and accelerations of the spherical joints of the moving platform over time. On the basis of these basic 
Table 6

Initial joint values of the 3RRPS S mechanism.

\begin{tabular}{|c|c|c|c|c|c|c|}
\hline \multirow[t]{2}{*}{ Joint } & \multicolumn{3}{|c|}{ Location (mm) } & \multicolumn{3}{|c|}{ Orientation } \\
\hline & $X$ & $Y$ & $Z$ & $X$ & $Y$ & $Z$ \\
\hline $\mathrm{A}$ & 731 & 525 & 1839 & 0.000 & 0.000 & 1.000 \\
\hline B & 296 & 205 & 1678 & 0.000 & 0.000 & 1.000 \\
\hline $\mathrm{C}$ & 421 & 590 & 1839 & 0.000 & 0.000 & 1.000 \\
\hline D & 614 & 529 & 1511 & 0.999 & 0.038 & 0.000 \\
\hline E & 715 & 92 & 1437 & 0.037 & 0.985 & 0.168 \\
\hline F & 251 & 268 & 2005 & 0.585 & 0.811 & 0.000 \\
\hline G & 40 & 21 & 1960 & 0.803 & 0.580 & 0.140 \\
\hline $\mathrm{H}$ & 339 & 593 & 1511 & 0.999 & 0.031 & 0.000 \\
\hline I & 408 & 156 & 1438 & 0.030 & 0.986 & 0.166 \\
\hline $\mathbf{J}$ & 689 & 594 & 1320 & & & \\
\hline $\mathrm{K}$ & 652 & 479 & 1839 & & N.A. & \\
\hline $\mathrm{L}$ & 386 & 547 & 1319 & & & \\
\hline M & 152 & 510 & 4311 & & & \\
\hline
\end{tabular}

kinematic characteristics, a number of additional kinematic performance indices are computed as well.

\subsection{Scraping}

The scraping capability of the earthmoving mechanism is evaluated in terms of the roll angle, which is defined with respect to the longitudinal axis of the SAE standard vehicle axis system, and represents the rotation around the vehicle driving direction. For the purpose of a comparative study between the 3RRPS-S, 3RRPS-UR and 3RRPS-RRR mechanisms, the roll angle is defined through the coordinates of spherical joints of the left and right lifting cylinders: $\gamma=\operatorname{atan}\left(\frac{y_{a}-y_{b}}{x_{a}-x_{b}}\right)-\operatorname{atan}\left(\frac{y_{a 0}-y_{b 0}}{x_{a 0}-x_{b 0}}\right)$

where $\left(x_{a}, y_{a}\right)$ and $\left(x_{b}, y_{b}\right)$ are the $X Y$ coordinates of the right and left lifting spherical joints, respectively. Coordinates $\left(x_{a 0}, y_{a 0}\right)$ and $\left(x_{b 0}, y_{b 0}\right)$ are their initial values. The roll angle is used to evaluate the kinematic performance, especially in the case of scraping.

Let us consider the new rods in the 3RRPS-UR and 3RRPS-RRR mechanisms in a vertical position. Different mechanisms can be derived based on the parametric length. Fig. 6 shows the roll angle of the 3RRPS-UR parallel mechanism with the rod length increasing from 200 to $600 \mathrm{~mm}$. The results show that the 3RRPS-UR mechanism presents a better scraping performance. Moreover, the scraping capability could be enhanced by increasing the rod length. The maximum roll angle is $32.43^{\circ}$ for a $600 \mathrm{~mm}$ rod length. If the upper rod length of the 3RRPS-RRR mechanism is varied from 200 to $400 \mathrm{~mm}$ and the lower one from 300 to $500 \mathrm{~mm}$, the roll angle can be derived as described in Fig. 7. This figure shows that the 3RRPS-RRR mechanism ensures the best scraping capability. Furthermore, the scraping capability is improved when short upper and lower rods are employed at the same time. The maximum roll angle equals $33.32^{\circ}$ when the $200 \mathrm{~mm}$ upper and $300 \mathrm{~mm}$ lower rods are chosen.

\subsection{Lifting}

The blade lifting height is adopted as a performance index to evaluate the blade lifting capability during the

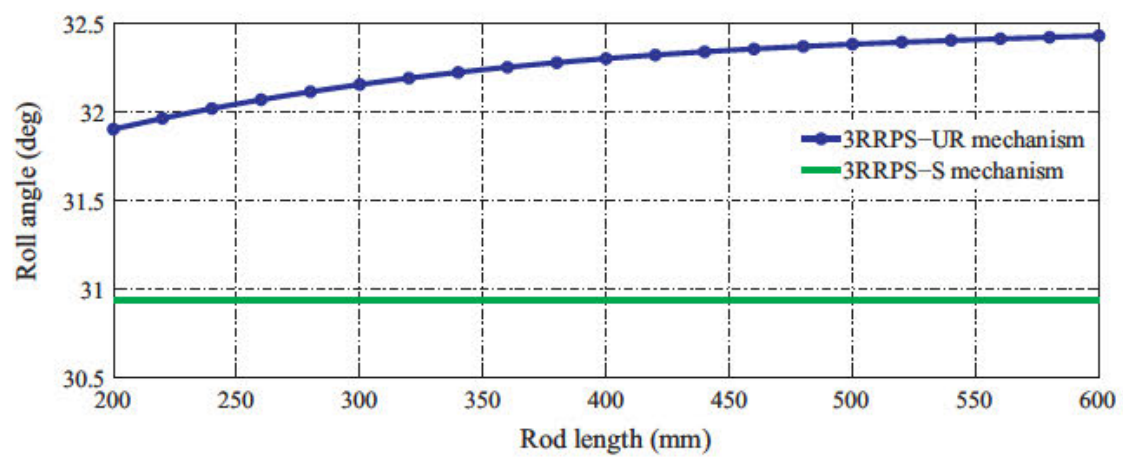

Fig. 6. Roll angle of the 3RRPS UR mechanism.

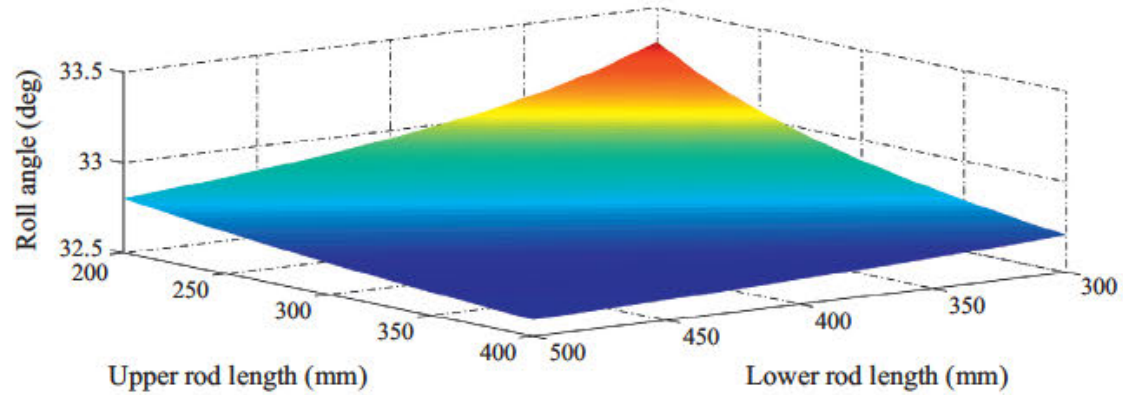

Fig. 7. Roll angle of the 3RRPS RRR mechanism. 


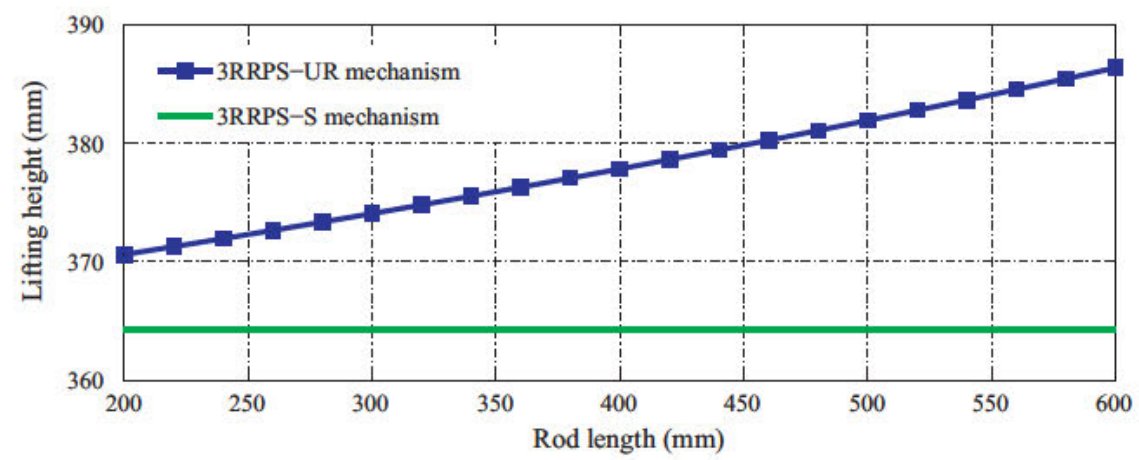

Fig. 8. Lifting height of the 3RRPS UR mechanism.

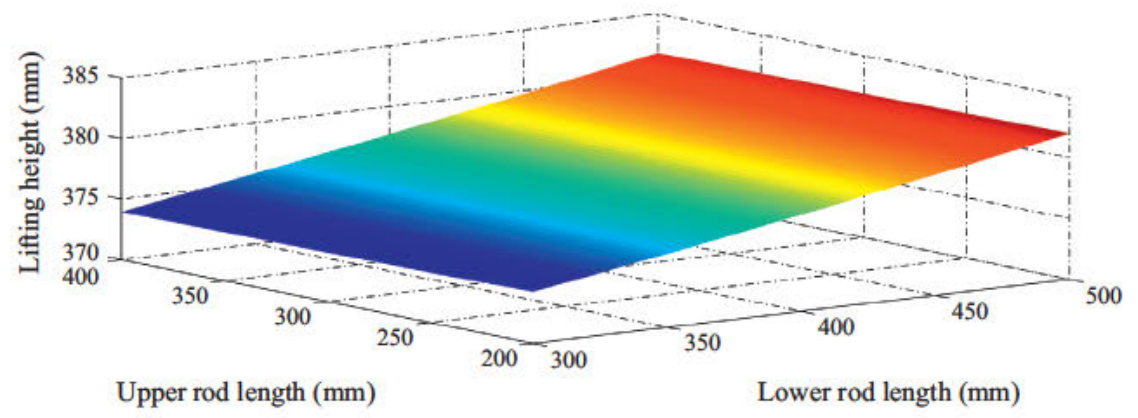

Fig. 9. Lifting height of the 3RRPS RRR mechanism.

earthwork, compaction and re-handling. The lifting height is closely related to the operation of blade lifting and rotation cylinders, thus the lifting capability is defined through the heights of the lifting cylinders' spherical joints:

$H=\frac{\left(y_{a}+y_{b}\right)}{2}-\frac{\left(y_{a 0}+y_{b 0}\right)}{2}$

Taking into account the parametric rods, which represent design variables in the 3RRPS-UR and 3RRPSRRR mechanisms, we can carry out a kinematic design process, as shown in Figs. 8 and 9, respectively. It can be seen that the 3RRPS-UR and 3RRPS-RRR mechanisms have a better lifting capability than the original 3RRPS-S mechanism. What is more, the lifting capability is improved by selecting longer rods. The maximum lifting height of the 3RRPS-UR mechanism equals $386.34 \mathrm{~mm}$ with a $600 \mathrm{~mm}$ rod. In the case of 3RRPS-RRR mechanism, it equals $382.02 \mathrm{~mm}$ with a $200 \mathrm{~mm}$ upper rod and a $500 \mathrm{~mm}$ lower one.

\subsection{Parallel lifting}

The parallel lifting capability is an important characteristic of blade performance. A better parallel lifting capability can reduce extra adjustment operations and suppress additional impacts on the system. The lifting heights of the spherical joints of the blade lifting cylinders are used to describe the blade parallel lifting capability:

$\Delta H=\left(y_{a}-y_{a 0}\right)-\left(y_{b}-y_{b 0}\right)$
The parallel lifting capability of the 3RRPS-UR and 3RRPS-RRR mechanisms in terms of the rod length is described in Figs. 10 and 11. Upon studying the results, it is clear that the parallel lifting capability of the 3RRPS-UR mechanism is somewhat worse than the original 3RRPS-S mechanism. The parallel lifting capability of the 3RRPS-RRR mechanism could be improved if appropriate rods were chosen.

Based on the analysis of scraping, lifting and parallel lifting, some conclusions can be drawn:

The 3RRPS-RRR mechanism has better kinematic performance than the original 3RRPS-S mechanism.

The 3RRPS-UR mechanism has better scraping and lifting capabilities than the original 3RRPS-S mechanism; the parallel lifting capability, in turn, is worse.

\subsection{Multi-objective optimization}

According to the previous analyses, increasing the rod length improves the lifting capability and deteriorates the parallel lifting capability. These two indices cannot reach maximum values simultaneously. It would therefore be desirable to carry out a multi-objective optimization process, which is widely used in vehicle systems (Callejo et al., 2015), to obtain optimal values of the lifting height and the parallel lifting capabilities at the same time. In such a multi-objective optimization problem, the length of the upper and lower rods would constitute the design variables. 


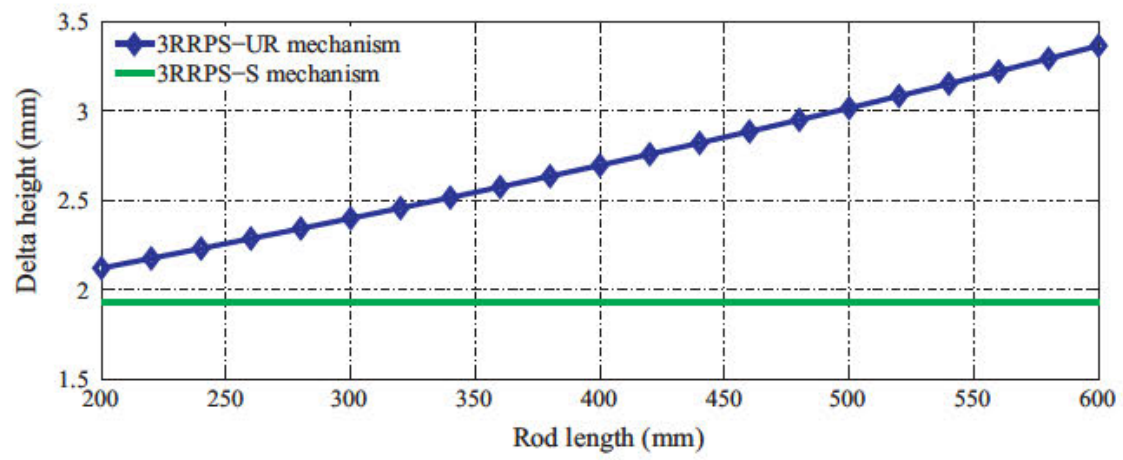

Fig. 10. Parallel lifting capability of the 3RRPS UR mechanism.

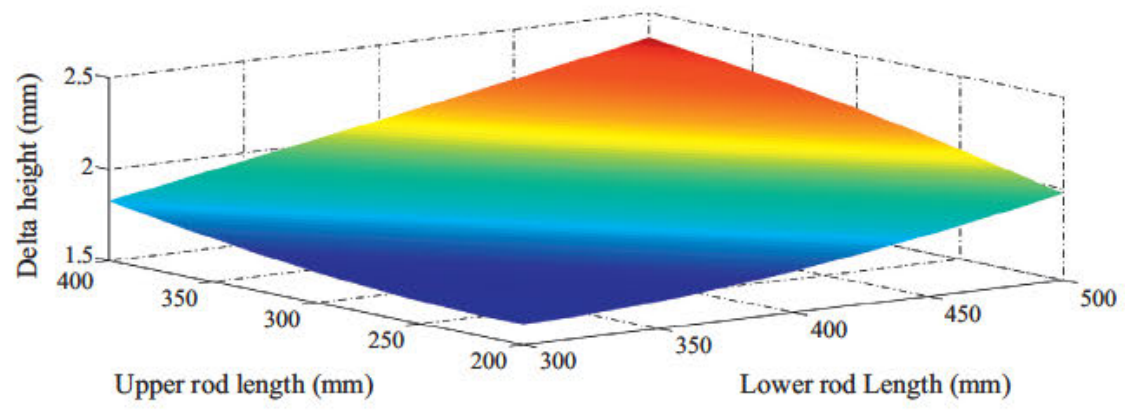

Fig. 11. Parallel lifting capability of the 3RRPS RRR mechanism.

The multi-objective optimization problem can be defined as:

$$
\begin{aligned}
& \max \mathbf{F}(\mathbf{x})=[H(\mathbf{x}),-\Delta H(\mathbf{x})]^{T} \\
& \text { s.t. }\left\{\begin{array}{l}
\mathbf{x} \in[\mathbf{b}, \mathbf{c}] \\
\mathbf{\Phi}(\mathbf{q}, t)=\mathbf{0} \\
\mathbf{q}=\mathbf{g}(\mathbf{x}, t)
\end{array}\right.
\end{aligned}
$$

where $\mathbf{x}=\left\{l_{1}, l_{2}\right\}^{T}$ is the vector of design parameters containing the upper rod length $\left(l_{1}\right)$ and lower rod length $\left(l_{2}\right)$; $\mathbf{F}(\mathbf{x})$ represents the vector of objective functions, namely the lifting height function $(H(\mathbf{x}))$ and parallel lifting function $(\Delta H(\mathbf{x})) ;[\mathbf{b}, \mathbf{c}]$ denotes the design domain. The Cartesian coordinates of each rod will be varied when a different rod length is selected, thus function $\mathbf{g}(\mathbf{x}, t)$ is introduced to express the system Cartesian coordinates in terms of the design parameters.

In previous sections, single-objective optimization processes were carried out to optimize the lifting height and parallel lifting capabilities, but these two objectives were evaluated in different dimensions. In order to implement a multi-objective optimization procedure in an easy way, fuzzy sets are introduced to measure the lifting height and parallel lifting capabilities in the same dimension and range (Zadeh, 1965; Gottwald, 2010). Specifically, two membership functions are introduced to transform each objective into a fuzzy set whose value ranges from zero to one. That way, the two objective functions can be evaluated along the same dimension. The membership functions can be written as follows:

$N_{1}(\mathbf{x})=\left[\frac{H(\mathbf{x})-H^{\circ}}{H^{*}-H^{\circ}}\right]^{k}, \quad k=1,2$
$N_{2}(\mathbf{x})=\left[\frac{\Delta H^{\circ}-\Delta H(\mathbf{x})}{\Delta H^{\circ}-\Delta H^{*}}\right]^{k}, \quad k=1,2$

where $H^{*}$ and $H^{\circ}$ are, respectively, the best and worst lifting height of the single-objective optimization procedure; $\Delta H^{*}$ and $\Delta H^{\circ}$ are the best and worst parallel lifting capabilities from the single-objective optimization; scalar $k$ denotes the membership function index.

These two objectives are then evaluated in the same dimension through the use of fuzzy sets and membership functions. A combined membership function, which evaluates the objectives simultaneously, is introduced by using weighting factors:

$D(\mathbf{x})=\sum_{i=1}^{n} w_{i} N_{i}(\mathbf{x}), \quad n=2$

$w_{1}+w_{2}=1$

$w_{1}=w_{2}$

where $w_{1}$ and $w_{2}$ are the weighting factors corresponding to the lifting height and parallel lifting, respectively. We assume that both objectives are equally relevant. This way, the multi-objective optimization problem can be transformed to a single-objective optimization problem 


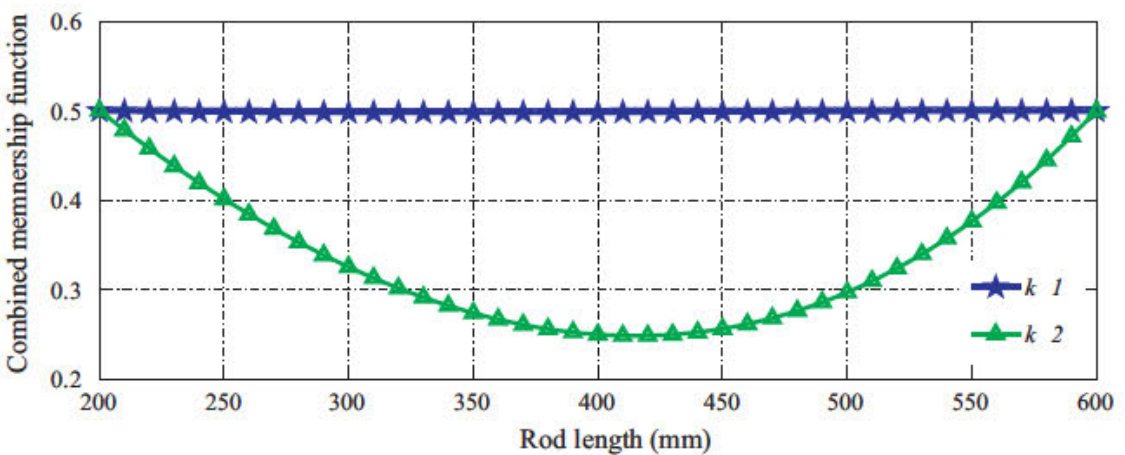

Fig. 12. Multi objective optimization results of the 3RRPS UR mechanism.

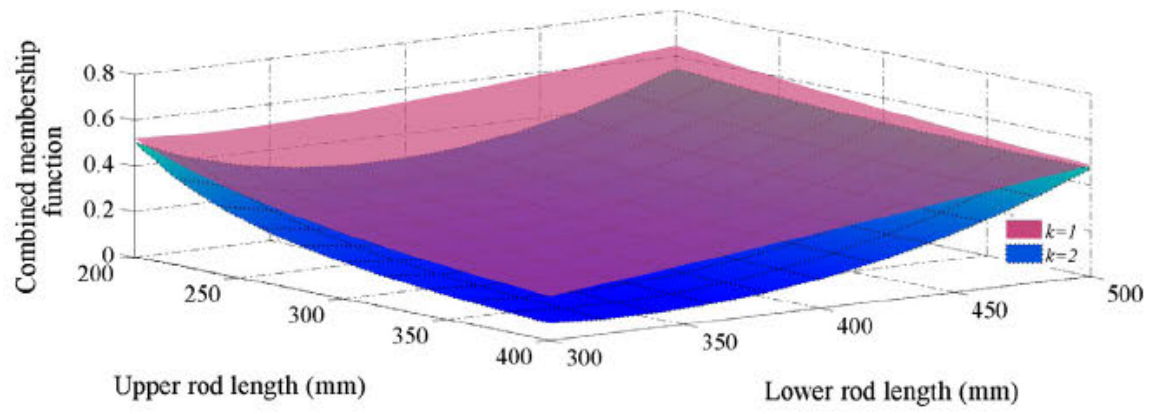

Fig. 13. Multi objective optimization results of the 3RRPS RRR mechanism.

Table 7

Multi objective optimization results.

\begin{tabular}{|c|c|c|c|c|c|c|c|c|}
\hline \multirow[t]{2}{*}{ Mechanism } & \multicolumn{2}{|c|}{ Membership function } & \multirow[t]{2}{*}{ Lifting height (mm) } & \multirow[t]{2}{*}{ Delta lifting height (mm) } & \multirow[t]{2}{*}{ Roll angle (deg) } & \multicolumn{3}{|c|}{ Rod length (mm) } \\
\hline & $k \quad 1$ & $k \quad 2$ & & & & $U$ & $S$ & $L$ \\
\hline 3RRPS S & N.A. & N.A. & 364.30 & 1.93 & 30.94 & & N.A. & \\
\hline 3RRPS UR & 0.49 & 0.50 & 370.59 & 2.12 & 31.90 & & 200 & \\
\hline 3RRPS RRR & 0.65 & 0.54 & 380.02 & 1.96 & 32.80 & 200 & & 500 \\
\hline
\end{tabular}

by introducing a combined membership function. The optimization problem can be re-defined as:

$$
\begin{array}{ll}
\max & D(\mathbf{x}) \\
\text { s.t. } & \left\{\begin{array}{l}
\mathbf{x} \in[\mathbf{b}, \mathbf{c}] \\
\mathbf{\Phi}(\mathbf{q}, t)=\mathbf{0} \\
\mathbf{q}=\mathbf{g}(\mathbf{x}, t)
\end{array}\right.
\end{array}
$$

Figs. 12 and 13 show the optimization results of the 3RRPS-UR and 3RRPS-RRR mechanisms, respectively. The lifting height and parallel lifting capabilities will reach optimal results simultaneously, as described in Table 7.

The same optimization results are obtained using different membership function indices according to Fig. 13 and Table 7. The optimal lifting height and delta lifting height of the 3RRPS-UR mechanism are $370.59 \mathrm{~mm}$ and $2.12 \mathrm{~mm}$, respectively. In contrast, the 3RRPS-RRR mechanism provides a better performance: $382.02 \mathrm{~mm}$ and
$1.96 \mathrm{~mm}$, respectively. Hence, we conclude that the 3RRPS-UR and 3RRPS-RRR mechanisms have better kinematic performance, and the 3RRPS-RRR mechanism provides the best comprehensive performance.

On the other hand, the delta lifting height of the optimized 3RRPS-RRR mechanism is $1.96 \mathrm{~mm}$, which is virtually the same as the original mechanism $(1.93 \mathrm{~mm})$. We can therefore say that they have the same parallel lifting capability. If one wants to obtain a better KPI performance, movable joints should be added to the design parameters and the design domain should be extended.

\section{Conclusions}

In this paper, a multibody model of an earthmoving mechanism has been investigated in detail, from the mechanism identification and innovative design to the kinematic analysis, all of which are rarely found in the literature. 
Through mechanism analysis and synthesis, the earthmoving system has been decomposed into a spatial parallel mechanism (3RRPS-S) and a spatial serial mechanism. We consider this a complex spatial hybrid mechanism. The 3RRPS-S mechanism, which carries out the blade rotation and lifting, is the key part of the grader's hybrid earthmoving mechanism. The spatial serial mechanism, in turn, is a combination of planar serial mechanisms, which control the blade lateral translation and cutting angle.

Afterwards, the 3RRPS-UR and 3RRPS-RRR spatial mechanisms have been presented, which are based on the original 3RRPS-S mechanism and include small-scale rods. These three mechanisms constitute a family of 3-DOF, 4 subchains, spatial parallel mechanisms. The kinematic performance of the mechanism family has been analyzed and compared. Some conclusions can be drawn:

The earthmoving device equipped with the 3RRPS-UR or 3RRPS-RRR mechanisms developed in the present work can provide better kinematic performance than the original 3RRPS-S mechanism.

The rods introduced in the 3RRPS-UR and 3RRPSRRR mechanisms can prevent fracture from happening in the traction and overhead frames.

The dynamic performance of the presented earthmoving mechanisms remains an open topic.

\section{Acknowledgments}

This study has been supported by the National Key Technology R\&D Program of China (Grant No. 2013BAF07B04) and the Ministry of Science and Innovation of Spain under Research Projects OPTIVIRTEST (TRA2009-14513-C02-01) and DOPTCARR (TRA201238826-C02-01). Financial support of the CSC Research Fellowship awarded to Yongjun Pan is also acknowledged.

\section{References}

Callejo, A., García de Jalón, J., Luque, P., Mántaras, D.A., 2015. Sensitivity based, multi objective design of vehicle suspension systems. J. Comput. Nonlinear Dynam. 10 (3), 031008.

Carmen, D., Silviu, N., 2012. Comparative analysis between operational performance of motor grader equipments. Ann. Univ. Dunarea de Jos Galati Fascicle XIV, Mech. Eng. 17 (2), 8790.

Chen, J., Qing, F., Pang, X., 2014. Mechanism optimal design of backhoe hydraulic excavator working device based on digging paths. J. Mech. Sci. Technol. 28 (1), 213222.

Choi, H. S., Han, C. S., young Lee, K., heon Lee, S., 2005. Development of hybrid robot for construction works with pneumatic actuator. Automat. Construct. 14 (4), 452459.

Fales, R., Kelkar, A., 2009. Robust control design for a wheel loader using and feedback linearization based methods. \{ISA\} Trans. 48 (3), 312 320.

Fox, B., Jennings, L., Zomaya, A., 2002. On the modelling of actuator dynamics and the computation of prescribed trajectories. Comput. Struct. 80 (7 8), 605614 .
Frimpong, S., Li, Y., 2005. Virtual prototype simulation of hydraulic shovel kinematics for spatial characterization in surface mining operations. Int. J. Surface Mining Reclamat. Environ. 19 (4), 238250.

Frimpong, S., Hu, Y., Awuah Offei, K., 2005. Mechanics of cable shovel formation interactions in surface mining excavations. J. Terramech. 42 (1), 1533 .

Frimpong, S., Hu, Y., Inyang, H., 2008. Dynamic modeling of hydraulic shovel excavators for geomaterials. Int. J. Geomech. 8 (1), 2029.

García de Jalón, J., 2007. Twenty five years of natural coordinates. Multibody Syst. Dynam. 18 (1), 1533.

García de Jalón, J., Callejo, A., 2011. A straight methodology to include multibody dynamics in graduate and undergraduate subjects. Mech. Machine Theory 46 (2), 168182.

García de Jalón, J., Unda, J., Avello, A., 1986. Natural coordinates for the computer analysis of multibody systems. Comput. Methods Appl. Mech. Eng. 56 (3), 309327.

Gottwald, S., 2010. An early approach toward graded identity and graded membership in set theory. Fuzzy Sets Syst. 161 (18), 23692379.

Hemami, A., 1992. Modelling, analysis and preliminary studies for automatic scooping/loading in a mechanical loader. Int. J. Surface Mining Reclamat. Environ. 6 (4), 151159.

Hemami, A., 1993. Modelling, analysis and preliminary studies for automatic scooping. Adv. Robot. 8 (5), 511529.

Jia, M., Ding, G., Qin, S., Li, R., He, Y., 2013. Research of design and analysis integrated information modeling framework for multibody mechanical system: with its application in the lhd design. Int. J. Adv. Manuf. Technol. 66 (9 12), 21072122.

Kim, Y.B., Ha, J., Kang, H., Kim, P.Y., Park, J., Park, F., 2013. Dynamically optimal trajectories for earthmoving excavators. Auto mat. Construct. 35, 568578.

Koivo, A.J., 1994. Kinematics of excavators (backhoes) for transferring surface material. J. Aerospace Eng. 7 (1), 1732.

Koivo, A.J., Thoma, M., Kocaoglan, E., Andrade Cetto, J., 1996. Modeling and control of excavator dynamics during digging opera tion. J. Aerospace Eng. 9 (1), 1018.

Li, Y., Frimpong, S., 2008. Hybrid virtual prototype for analyzing cable shovel component stress. Int. J. Adv. Manuf. Technol. 37 (5 6), 423 430.

Li, Y., Wang, J., Liu, X. J., Wang, L. P., 2010. Dynamic performance comparison and counterweight optimization of two 3 DOF parallel manipulators for a new hybrid machine tool. Mech. Machine Theory 45 (11), 16681680.

Rusiński, E., Moczko, P., Czmochowski, J., 2008. Numerical and experimental analysis of a mine's loader boom crack. Automat. Construct. 17 (3), 271277.

Seungjin, Y., Cheol Gyu, P., Bokman, L., Lee, K.I., Park, F.C., 2010. Bandwidth maximizing design for hydraulically actuated excavators. J. Vib. Control 16 (14), 21092130.

Shen, J., Wang, G., Bi, Q., Qu, J., 2013. A comprehensive genetic algorithm for design optimization of $\mathrm{Z}$ bar loader working mechanism. J. Mech. Sci. Technol. 27 (11), 33813394.

Sobczyk, A., Tora, G., 1998. Grader blade stabilization system. Automat. Construct. 7 (5), 385389.

Staicu, S., 2009. Dynamics of the spherical 3 UPS/S parallel mechanism with prismatic actuators. Multibody Syst. Dynam. 22 (2), 115132.

Staicu, S., Liu, X. J., Wang, J., 2007. Inverse dynamics of the half parallel manipulator with revolute actuators. Nonlinear Dynam. 50 (1 2 ), 1 12.

Takahashi, H., Morikawa, Y., Tateyama, K., Fukagawa, R., 2004. Study on the mechanism of over head type load haul dump with a vessel. J. Terramech. 41 (2 3), 175185 .

Towarek, Z., 2003. Dynamics of a single bucket excavator on a deformable soil foundation during the digging of ground. Int. J. Mech. Sci. 45 (6 7), 10531076.

Vaha, P.K., Skibniewski, M.J., 1993. Dynamic model of excavator. J. Aerospace Eng. 6 (2), 148158. 
Wu, J., Wang, J., Wang, L., Li, T., 2009. Dynamics and control of a planar 3 DOF parallel manipulator with actuation redundancy. Mech. Machine Theory 44 (4), 835849.

Wu, J., Li, T., Wang, J., Wang, L., 2013. Stiffness and natural frequency of a 3 DOF parallel manipulator with consideration of additional leg candidates. Robot. Autonom. Syst. 61 (8), 868875.

Xu, X., Huang, Z., 1985. Earthmoving Machine Design. China Machine Press.
Yen, P. L., Lai, C. C., 2009. Dynamic modeling and control of a 3 DOF cartesian parallel manipulator. Mechatronics 19 (3), 390398.

Zadeh, L., 1965. Fuzzy sets. Inform. Control 8 (3), 338353.

Zhen, J., Pan, C., Jangale, A., Salisbury, B., 2011. Identification and reduction of booming noise on a motor grader. SAE Technical Paper 2011011729. 